Jonathan Rappe

Université de Liège

Patricia Schillings

Université de Liège

Yves Depluvrez

Université de Liège

Charlotte Dejaegher

Université de Liège

\section{Analyse du modèle opératif d'un enseignant en deuxième année primaire dans le cadre de I'enseignement de la lecture}

\author{
Analysis of a teacher's operative model in second \\ grade in the context of teaching how to read
}

\section{ésumé}

Cette étude vise à décrire le modèle opératif d'une enseignante de deuxième grade pour l'enseignement de la lecture. À la suite d'observations et d'entretiens, ce modèle a été schématisé afin de déterminer l'influence des concepts organisateurs dans quatre classes de situations : I'enseignement de la compréhension, l'enseignement des correspondances graphophonémiques, le recours à la lecture oralisée et I'usage de l'écriture dans des tâches de lecture. Nous postulons ensuite l'existence de freins conceptuels à la mise en œuvre de pratiques efficaces. Par exemple, I'un des concepts utilisés par le sujet semble entraver l'articulation de l'enseignement de la lecture et de l'écriture.

\section{Mots-clés}

Enseignement de la lecture, modèle opératif, concepts organisateurs.

Abstract

This study aims to describe the operative model of a second grade teacher in the context of the teaching of reading. Following observations and interviews, this operative model was schematized in order to highlight how the teacher's pragmatic concepts potentially influence her activity in four classes of situations: teaching text comprehension, teaching grapho-phonemic correspondences, the emphasis on oral reading and the use of writing in the teaching of reading. We then postulate the existence of conceptual obstacles to the implementation of effective teaching practices. For example, a concept used by the subject seems to impede the joint teaching of reading and writing.

Keywords

Teaching reading, operative model, organizers of the teaching activity.

\section{Contexte}

En Belgique francophone, les enquêtes internationales indiquent que les performances en lecture des élèves de primaire sont globalement inférieures à la moyenne des pays comparables (Mullis, Martin, Foy et Hopper, 2017; Schillings, Géron et Dupont, 2017; Schillings, Hindryckx, Dupont, Matoul et Lafontaine, 2012; Schillings et Lafontaine, 2013). Les résultats de l'étude internationale PIRLS 2011 comme ceux issus du cycle 2016 montrent qu'en Fédération WallonieBruxelles (FW-B), les processus de compréhension de haut niveau de complexité apparaissent nettement moins bien maitrisés en quatrième année primaire (grade 4) que dans d'autres systèmes éducatifs où les élèves de même niveau scolaire et/ou de même âge sont en moyenne plus nombreux à atteindre les niveaux de compétences les plus experts (Mullis et al., 2017; Schillings, Dupont, Géron et Matoul, 2017).

Les réponses recueillies auprès des enseignants lors de cette même étude PIRLS 2011 ont permis de comparer les activités pédagogiques que les enseignants belges proposent à leurs élèves de quatrième année primaire $(\mathrm{CM} 1)$ à celles mises en place par des enseignants issus des pays ayant obtenu des performances supérieures à la moyenne internationale (Schillings, Géron et al., 2017). Parmi les disparités mises en évidence, on note chez les enseignants belges un recours moins fréquent aux activités visant des compétences de lecture plus complexes (telles qu'interpréter et intégrer des idées et des informations), une tendance moins accrue à mettre en place des pratiques de lecture impliquant des échanges avec les pairs ou avec le texte via un écrit personnel, ainsi qu'une tendance à faire lire aux élèves des textes de moins grande envergure (par exemple, des récits courts plutôt que des livres divisés en chapitres). Plus globalement, on observe que dans certains systèmes éducatifs anglophones tels que ceux de l'Ontario ou de l'Angleterre où les élèves présentent 
des performances moyennes élevées, les processus de compréhension de bas niveau (centrés sur le traitement du contenu du ou des textes) et de haut niveau (qui sollicitent davantage d'élaboration de la part du lecteur) sont travaillés de manière conjointe et articulés dès le premier cycle, alors qu'ils sont abordés de manière successive, selon une logique allant du simple au complexe, dans les systèmes francophones et en particulier en FW-B (Schillings, Géron et al., 2017).

Si un nombre conséquent de méta-analyses, couplées aux résultats des enquêtes internationales, attestent de l'efficacité de certaines pratiques d'enseignement de la lecture, les recommandations issues de ces diverses recherches ne semblent exercer qu'une faible influence sur les pratiques effectives des enseignantes (Goigoux, 2007; Prost, 2001) et, au vu des résultats PIRLS, particulièrement en FW-B.

Qu'ils partagent ou non la conviction du bien-fondé de ces recommandations, la seule présentation de ces recommandations aux enseignants ne peut en effet suffire. En effet, selon leur expérience antérieure, leurs croyances et connaissances, la redéfinition de la tâche d'enseignement de lecture réalisée par ces professionnels différera de la tâche prescrite (Leplat, 2011). Le caractère situé (Vergnaud, 1996), pluriel (Goigoux et Cèbe, 2009) et multifinalisé (Goigoux, 2007) de l'activité d'enseignement rendent d'autant plus variable la mise en ouvre effective de ces recommandations.

Dès lors, à l'instar des travaux de Lefeuvre et Murillo (2017) en France, il semble pertinent de s'intéresser à l'activité effective d'enseignement de la lecture des instituteurs primaires de FW-B et de tenter d'identifier les conceptions et les conceptualisations qui orientent et guident cette activité afin de mieux comprendre les écarts observés entre les pratiques des enseignants et celles mises en avant par la recherche et les résultats des enquêtes internationales. Dans cette visée, notre étude vise à décrire le modèle opératif d'une enseignante de deuxième année primaire (CE1), construit à la suite d'observations filmées et d'entretiens d'explicitation. Cette analyse permettra ensuite d'identifier ce qui, dans les croyances et concepts organisateurs de l'action de l'enseignante, pourrait constituer un frein à la mise en œuvre de certaines démarches ayant montré leur efficacité dans d'autres systèmes éducatifs.

\section{Cadre conceptuel}

\section{La conceptualisation dans l'action}

Principe fondateur de la théorie de la conceptualisation dans l'action (Vergnaud, 1996), le décalage entre la réussite et la compréhension, mis en évidence par Piaget (1974) amène à une première conclusion : l'action est une connaissance autonome. À sa suite, Vergnaud (1996) postulera que la connaissance se présente sous deux formes : la forme prédicative et la forme opératoire. La forme prédicative permet au sujet de comprendre le réel et ses constituants tandis que la forme opératoire lui permet d'agir dans le réel, de s'y adapter ou de l'adapter. Ces deux aspects sont interdépendants et se soutiennent mutuellement (Pastré, Mayen et Vergnaud, 2006).

Selon Vergnaud (1996), le schème représente « une organisation invariante de l'activité pour une classe de situations données". Ainsi, l'action d'un individu en situation professionnelle est régie par des conceptions qui guident et orientent son activité. Il s'agit des concepts pragmatiques (Pastré, 2011) qui peuvent être implicites, inconscients pour le sujet ou, au contraire, explicites. À titre d'exemple, 
l'étude de Lefeuvre et Murillo (2017), ciblée sur l'analyse de l'activité de découvertes collectives de textes menée par une enseignante de $\mathrm{CP}$, a mis en évidence le rôle joué par un concept pragmatique dans l'ajustement de son activité d'enseignement. À partir du troisième trimestre de l'année scolaire, l'enseignante introduit des objectifs différenciés (lecture autonome pour les élèves forts et lecture menée sous la guidance de l'enseignante pour les autres) lorsqu'elle se rend compte, d'une part, que les élèves faibles et moyens ne suivent pas (avec pour indicateurs que les élèves se désintéressent de la tâche, que certains deviennent indisciplinés et qu'elle doit formuler de nombreux rappels à l'ordre) et, d'autre part, que les élèves forts, eux, s'ennuient (avec pour indicateurs qu'ils «s'impatientent » et qu'elle doit réfréner leurs interventions au risque qu'ils se désengagent de la tâche). Lefeuvre et Murillo (2017) considèrent que « l'écart entre le niveau de difficulté de la tâche et les performances des élèves » est un concept pragmatique utilisé inconsciemment par l'enseignante pour guider son activité d'enseignement de la lecture.

Ces concepts qui orientent l'activité des professionnels peuvent être de deux origines : soit ils sont directement construits par le professionnel dans l'action (on parle alors de concepts pragmatiques), soit ils sont issus de savoirs scientifiques ou techniques. On parle alors de concepts pragmatisés (Pastre囚, 2011). Selon Pastré (2011), chaque concept pragmatique est associé à une ou plusieurs classes de situations. Dans l'action, l'individu prendrait appui sur des indicateurs pour diagnostiquer une situation, et ainsi mobiliser un concept pragmatique plutôt qu'un autre pour orienter son action.

L'ensemble de ces concepts pragmatiques et de leurs indicateurs constitue ce que Pastré définit comme une «structure conceptuelle». Indispensable pour permettre au professionnel de prélever les informations nécessaires afin de faire un diagnostic de la situation, elle lui permet d'orienter efficacement son action (Pastré, 2013). Le modèle opératif, propre à chaque professionnel, représente quant à lui « la manière dont cet acteur s'est approprié plus ou moins bien, plus ou moins complètement, la structure conceptuelle d'une situation» (Pastré, 2013, p. 179). Appliquée au domaine de l'enseignement de la lecture, l'étude des modèles opératifs de professionnels donne à voir les aspects du réel pris en compte dans l'action et les savoirs d'action qui fondent leur activité.

\section{Une structure conceptuelle de l'enseignement de la lecture?}

Bien que plusieurs travaux s'y soient déjà intéressés, l'élaboration d'une structure conceptuelle dans le domaine de l'enseignement est, selon Vinatier (2013), si pas impossible, du moins complexe. En effet, l'analyse de l'activité de l'enseignant ne peut se départir de la prise en compte de celle des élèves et du processus interactionnel qui les influence réciproquement.

Dès lors, nous ne tenterons pas d'établir une telle structure conceptuelle pour l'enseignement de la lecture. En revanche, sans chercher à en dresser une liste exhaustive, nous présenterons dans ce point certaines recommandations pour l'enseignement de la lecture issues de pratiques épinglées comme efficaces par différentes recherches ou méta-analyses.

Dans l'étude française Lire-Écrire menée par Goigoux, Jarlégan et Piquée (2015) dans 131 classes de $\mathrm{CP}$, des observations de pratiques d'enseignement de la lecture ont été menées. Cette étude française ne conclut pas à une supériorité attestée d'une approche d'enseignement de la lecture sur une autre. L'auteur postule que c'est une combinaison complexe de variables qui contribue à l'efficacité 
de l'enseignement (étude du code, enseignement de la compréhension de l'écrit, enseignement de l'écriture, étude de la langue, lecture à haute voix, mais aussi climat de classe, engagement dans le travail, recours aux rétroactions immédiates, explicitation des démarches cognitives, utilisation efficace du temps d'interaction avec un adulte...).

Ces résultats convergent avec les recommandations pratiques fondées sur les études scientifiques anglophones ayant fourni des preuves de leur efficacité. Parmi les recommandations que Higgins, Henderson, Martell, Sharples et Waugh (2016) associent à un enseignement efficace de la lecture, le développement conjoint du langage oral, de la lecture et de l'écriture est préconisé sous le terme de littératie. Selon ces auteurs, la mise en place d'une démarche d'enseignement de la lecture qui conjugue l'apprentissage du décodage et des habiletés de compréhension, l'enseignement systématique des correspondances grapho-phonémiques de même que l'enseignement de stratégies leur permettant de planifier et de réguler leurs écrits figurent parmi les recommandations didactiques fondées sur la preuve scientifique.

\section{Problématique de recherche}

Au vu de ces différents constats, deux questions se posent :

1. Quels sont les concepts organisateurs qui guident l'activité de l'enseignante observée lorsqu'elle enseigne la lecture?

2. Peut-on identifier, dans ce modèle, des leviers ou, au contraire, des freins conceptuels à un enseignement efficace de la lecture?

\section{Méthodologie}

La présente recherche s'inscrit dans une démarche de recherche clinique (Clot et Leplat, 2005). Nous avons dès lors tenté de respecter les trois principes qui fondent cette approche : considérer l'objet d'étude dans sa globalité, tenter d'examiner l'objet dans toute sa complexité et accorder un intérêt particulier au rôle du sujet.

\section{Participants}

Cette étude a été conduite dans le cadre d'un mémoire de fin de master en sciences de l'éducation (Rappe, 2015).L'échantillon initial était composé de quatre enseignants. Le recrutement de ces derniers s'est effectué sur une base volontaire. Plusieurs écoles de la province de Liège ont été contactées et les quatre enseignants retenus sont ceux ayant accepté de prendre part au projet de recherche. Pour mieux convenir au format d'une contribution comme celle-ci, nous avons décidé de ne conserver qu'un seul sujet $^{1}$ (Julie). Cette enseignante exerce en deuxième année primaire et possède six ans d'expérience dans l'enseignement. Sa classe compte 19 élèves. Par ailleurs, il est à noter qu'elle garde le même groupe classe en première et en deuxième année. 


\section{Instrumentation}

Deux types d'outils ont été utilisés pour mener à bien cette étude : des observations filmées de séquences d'enseignement/apprentissage menées par le sujet, suivies d'entretiens individuels. Ces collectes en deux étapes ont été réalisées à cinq reprises, à raison d'une fois par mois pendant cinq mois. Par ailleurs, le choix des séances observées était laissé à l'enseignant. Il lui était en effet demandé de sélectionner un ensemble de cinq séances représentant au mieux, selon lui, sa pratique d'enseignement de la lecture.

Les séquences observées s'étalaient sur 30 à 50 minutes. Elles étaient entièrement filmées. L'objectif de ces observations était de capter toute la complexité de la situation observée, et d'en garder une trace observable comme support à l'entretien qui lui faisait suite. En plus de cet enregistrement, le chercheur prenait des notes sur les éléments de l'action de l'enseignant à propos desquels le chercheur avait besoin d'informations supplémentaires (par exemple, le recours à un référentiel ou le rappel d'une leçon précédente).

L'entretien mené après chaque séquence observée visait à faire revenir l'enseignant sur le déroulement de son activité. À cette fin, nous avons eu recours à la démarche d'entretien d'explicitation (Vermersch, 2004). Cette méthode nous semble être la plus pertinente au vu des éléments investigués chez le sujet, à savoir les concepts organisateurs de son action professionnelle. En effet, l'entretien d'explicitation a pour finalité d'amener le sujet à décrire sa propre action, de l'aider à en prendre conscience, à la conceptualiser, et à verbaliser cette conceptualisation. Pour le chercheur, l'entretien permet d'entrevoir le système interprétatif du sujet et d'avoir accès à une série d'informations sur son fonctionnement et ses représentations. Le chercheur peut alors, au travers de l'analyse des entretiens (voir section suivante), tenter d'inférer les concepts organisateurs de l'action du sujet.

Dans la pratique, cette technique d'entretien requiert de ne pas envisager à l'avance des questions à poser au sujet, mais de permettre à ce dernier de revenir aisément sur son action au moyen d'un observable (dans notre cas, l'enregistrement de la séquence d'enseignement). Néanmoins, certaines lignes directrices ont été respectées dans le cadre de notre collecte de données. D’abord, en début d'entretien, nous proposions systématiquement au sujet de revenir librement, s'il le désirait, sur un ou plusieurs moments de son choix dans la séquence observée. Cette étape visait à lui permettre de se replonger spontanément dans leur action, ce que Vermersch (2004) appelle une "visée à vide ». Ensuite, l'enregistrement de la séquence était visionné avec le sujet et le chercheur posait diverses questions l'invitant à expliciter son action et les raisons de cette dernière. Enfin, il est important de noter que nous cherchions à obtenir un niveau de détails suffisant dans les explications du sujet pour rendre l'action de celui-ci suffisamment intelligible.

\section{Analyse des données}

Les entretiens menés avec les quatre sujets de l'étude ont fait l'objet d'une analyse de contenu proche de celle de L'Écuyer (1987), mais adaptée à nos objectifs de recherche. En voici les différentes étapes :

\section{Retranscription des entretiens.}

2. Lecture des entretiens afin de sélectionner les classes de situations sur lesquelles les sujets semblaient plus spécifiquement se centrer. Cette sélection a reposé à la fois sur leur occurrence 
et sur l'importance que le sujet semblait leur accorder (la place qu'elles occupaient dans la discussion, l'importance déclarée par le sujet, le niveau de détail qu'il proposait). Quatre d'entre elles ont été retenues (voir section suivante).

3. Catégorisation et étiquetage des portions de discours selon les concepts organisateurs explicitement évoqués ou semblant transparaitre dans les propos du sujet à l'intérieur des sections d'entretien conservées (correspondant aux quatre classes de situations sélectionnées).

4. Mise en lien des différentes portions d'entretien pour tenter d'identifier les concepts organisateurs principalement utilisés, les vérifier, les clarifier et les nommer. C'est aussi lors de cette étape que les indicateurs associés à chacun des concepts ont été identifiés.

5. Élaboration des modèles opératifs des enseignants : cette modélisation intègre les quatre classes de situations retenues ainsi que les concepts organisateurs et leurs indicateurs (voir cidessous).

\section{Résultats}

L'analyse des entretiens menés avec les quatre sujets conduit à identifier les classes de situation d'enseignement de la lecture apparaissant les plus saillantes dans leurs verbalisations. Comme mentionné ci-dessus, quatre d'entre elles ont été sélectionnées : l'enseignement de la compréhension de textes, l'enseignement des correspondances grapho-phonémiques, la place accordée à la lecture oralisée et l'usage de l'écriture dans l'enseignement de la lecture ${ }^{2}$. Ce découpage qui résulte des verbalisations du sujet ne doit pas occulter les interactions entre ces classes de situations. En effet, lors des séances observées, plusieurs de ces classes de situations étaient généralement mises en œuvre. Le tableau cidessous reprend les séquences observées et les classes de situations rencontrées.

\section{Tableau 1}

Objets principaux des leçons observées chez Julie et classes de situations associées

\begin{tabular}{|c|c|c|c|c|c|}
\hline & Leçon 1 & Leçon 2 & Leçon 3 & Leçon 4 & Leçon 5 \\
\hline 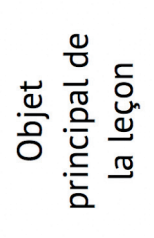 & $\begin{array}{l}\text { L'inférence (au } \\
\text { travers d'un } \\
\text { texte } \\
\text { descriptif) }\end{array}$ & $\begin{array}{l}\text { L'inférence (au } \\
\text { travers d'un } \\
\text { texte narratif) }\end{array}$ & $\begin{array}{l}\text { Association de } \\
\text { couvertures de } \\
\text { livres aux } \\
\text { synopsis } \\
\text { correspondants }\end{array}$ & $\begin{array}{l}\text { Le phonème } \\
\text { "è" }\end{array}$ & Lecture rapide \\
\hline 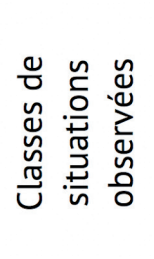 & $\begin{array}{l}\text { Compréhension } \\
\text { Usage de } \\
\text { l'écriture } \\
\text { Lecture oralisée }\end{array}$ & $\begin{array}{l}\text { Compréhension } \\
\text { Lecture oralisée } \\
\text { Correspondances } \\
\text { grapho- } \\
\text { phonémiques }\end{array}$ & $\begin{array}{l}\text { Compréhension } \\
\text { Lecture oralisée }\end{array}$ & $\begin{array}{l}\text { Correspondances } \\
\text { grapho- } \\
\text { phonémiques }\end{array}$ & $\begin{array}{l}\text { Compréhension } \\
\text { Correspondances } \\
\text { grapho- } \\
\text { phonémiques } \\
\text { Usage de } \\
\text { l'écriture }\end{array}$ \\
\hline
\end{tabular}


1. Quels sont les concepts organisateurs qui guident l'activité de l'enseignante lorsquelle enseigne la lecture?

Dans ce point, le modèle opératif est d'abord schématisé afin d'illustrer l'influence potentielle des concepts pragmatiques mobilisés sur les quatre classes de situations retenues. Ensuite, les concepts, ainsi que les autres éléments de leur modèle opératif, sont expliqués et illustrés par des extraits d'entretien menés à la suite des cinq leçons dont l'objet est détaillé dans le tableau 1 ci-dessus.

\section{Modèle opératifdu sujet}

Dans la modélisation (figure 1), les classes de situations sont représentées par de grands carrés blancs juxtaposés. Les concepts pragmatiques sont symbolisés par des formes ovoïdes de couleur. Leur emplacement sur le modèle donne une indication sur la ou les classes de situations dans lesquelles ils semblent être utilisés. Les indicateurs sur lesquels Julie porte son attention pour diagnostiquer et réguler son activité apparaissent dans des cadres reliés au concept correspondant.

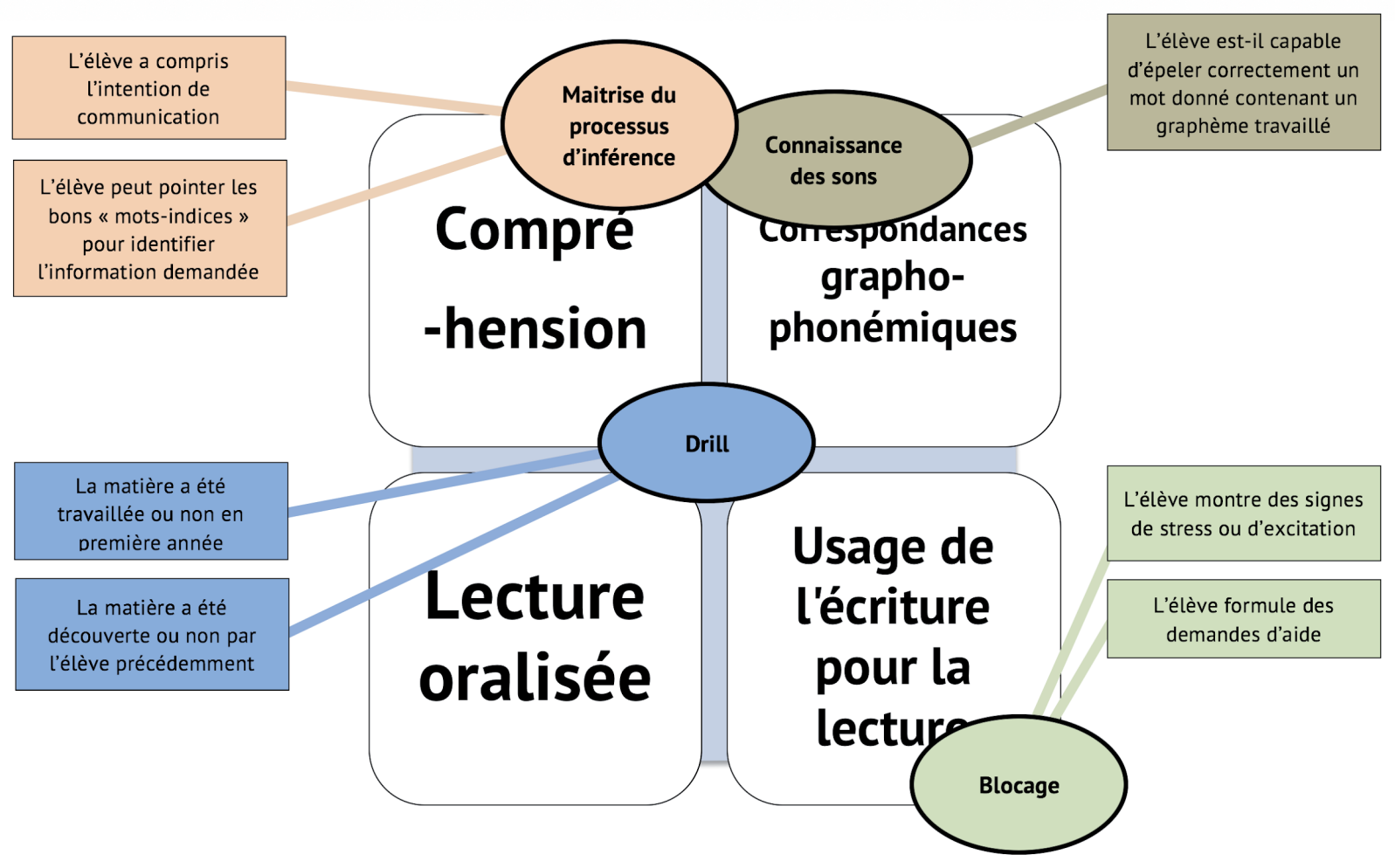

Figure 1

Modèle opératif de Julie 
Le concept de maitrise du processus d'inférence apparait comme un des concepts organisateurs du modèle opératif de ce sujet. Un exemple concret issu d'une activité de lecture collective (sur la base d'un texte uniquement narré en « je ", leçon 2 dans le tableau 1) illustre le rôle joué par ce concept dans la régulation de son activité. Lorsquelle constate que certains enfants se méprennent sur l'identité du narrateur, l'enseignante demande aux enfants d'identifier les "mots-indices ${ }^{3}$ contenus dans le texte afin qu'ils réussissent à déterminer qui en était le narrateur. En effet, lors du deuxième entretien, elle déclare : «ils étaient attachés à l'idée que ceétait un petit garçon, ils voulaient se persuader que c'était bien ça quoi, ils ne voulaient pas changer d'idée. [...] l'enfant part sur une idée et c'est pas pour ça que c'est forcément la bonne » ( $2^{\mathrm{e}}$ entretien). Ainsi, en tenant compte des termes « maman » et " galoper », l'enfant pouvait déterminer, par inférence, que le narrateur était un poulain.

La maitrise du processus "d'inférence ${ }^{4}$ est donc un concept qui organise l'action de l'enseignante. Il est mobilisé via le recours à deux indicateurs principaux : la compréhension générale par l'élève de l'intention de communication (principalement ici l'identification du narrateur) et l'utilisation par l'élève du contexte d'un mot pour en identifier la signification (l'élève a-t-il pointé les bons " motsindices » pour identifier l'information demandée?). Au cours des entretiens, l'enseignante explique les mots-indices: " oui, c'est déjà ce quion avait fait avec la description où ils devaient retrouver les mots-indices qui leur permettaient de dire que c'était ce monstre-là et pas un autre " ( $3^{\mathrm{e}}$ entretien).

Un deuxième concept de connaissance des sons permet à l'enseignante de guider son action (Pastré et al., 2006) dans la classe de situations ciblée sur l'étude des correspondances grapho-phonologiques. Lors des séances de lecture collective, afin de vérifier leur maitrise et connaissance des «sons ", elle a pour habitude de demander à certains élèves d'épeler un mot donné contenant un graphème travaillé. Selon elle, elle vérifie la "connaissance des sons » en observant si l'élève est capable ou non d'épeler le mot correctement.

En fonction de son diagnostic de situation, l'enseignante oriente son action : si l'enfant reconnait sans difficultés le "son", alors elle estime qu'elle peut lui proposer une phase d'exercisation, quelle qualifie de "drill ». C'est ce que montre cet extrait du deuxième entretien : " [le son] "au" tu vois, ils le connaissent. Donc, c'est plus, ça va être plus maintenant de l'exercice de drill qu'autre chose que... qu'une vraie découverte ». Dans le cas contraire (si l'élève ne reconnait pas le «son »), l'enseignante cherche à proposer des mises en situation, qu'elle nomme "découverte » : elle propose un texte présentant une forte occurrence du phonème étudié.

Par ailleurs, il est ressorti du discours de Julie qu'elle considère que la maitrise des différents " sons » d'un texte et la compréhension globale de ce dernier sont en relation. Par exemple, lors d'une activité observée (leçon 5 dans le Tableau 1), l'enseignante a proposé aux élèves de lire un texte dont certains mots étaient lacunaires (elle avait délibérément retiré certains graphèmes). Les enfants avaient pour tâche à la fois de comprendre le texte, et de retrouver les graphèmes manquants : "Ça, c'est de la lecture technique, bien que je trouve aussi que c'est de la compréhension de lecture [...] puisqu'il faut quand même comprendre le mot pour savoir quel son le complète" ( $5^{\mathrm{e}}$ entretien). Ainsi, il semble que, dans certaines situations, le concept de «connaissance des sons » se rapprochait du travail sur la compréhension, ce qui justifie la position du concept dans la modélisation. 
Un troisième concept pragmatique semble jouer un rôle essentiel dans l'action de Julie : le concept de drill. Chez elle, ce concept se définit comme l'entrainement ou l'exercisation des élèves dans les domaines qui ont déjà fait l'objet d'un apprentissage en première année. Pour bien comprendre ce concept, il est important de rappeler que Julie suit son groupe-classe en deuxième année. Ce concept s'opérationnalise via des indicateurs liés à la progression des contenus tels que : cette matière a-t-elle été découverte en première année? A-t-elle déjà été travaillée récemment par les élèves? Contrairement au concept de connaissance des sons, qui concerne une classe de situations spécifique de la tâche d'enseignement de la lecture, celui de drill semble opérer dans les quatre classes de situations retenues (cf. Figure 1). Les extraits suivants illustrent ce concept dans les classes sélectionnées.

D'abord, l'enseignement de la compréhension : "la compréhension de textes [amène à] beaucoup les entrainer à lire, à faire de l'inférence. C'est un peu ça le but en deuxième année, c'est vraiment de les "driller" à faire ça» ( $2^{\mathrm{e}}$ entretien). Ensuite, l'enseignement des correspondances grapho-phonémiques : "Donc, ils ont un manuel de lecture avec des textes vraiment ouù on retrouve plein de "e". On va refaire des exercices là-dessus, du drill encore un petit peu. Ce riest plus comme en première année où le son, ça se voit pendant une semaine » ( $2^{\mathrm{e}}$ entretien). Et, enfin, la lecture oralisée : «ben oui pour qu'ils lisent à haute voix, surtout qu'on nous encourage à le faire et donc voilà, je trouve que [les faire lire chacun à leur tour] est un bon moyen " ( $3^{\mathrm{e}}$ entretien).

Un quatrième et dernier concept pragmatique a pu être identifié lors des retours sur l'activité : le concept de blocage. Il concerne plus spécifiquement l'usage de l'écriture dans l'enseignement de la lecture. Selon l'enseignante, l'écriture par l'enfant d'un mot dont il ne connaît pas la graphie le « bloque ». Pour diagnostiquer la présence de ce blocage dans une situation de classe, les indicateurs qu'utilise l'enseignante sont l'état émotionnel perçu de l'enfant (est-ce qu'il semble stressé?) et la fréquence des demandes d'aide formulées par celui-ci.

Dans l'exemple qui suit, lors d'une tâche d'écriture qui vise le développement de la lecture (proposée dans la foulée de la leçon 5, voir le tableau 1), on observe que ce concept organise son activité. L'enseignante proposait en effet aux enfants d'écrire une phrase racontant ce qu'ils ont fait lors de leur weekend et de l'afficher au tableau afin que les pairs puissent lire leur production écrite : "Oui, oui, souvent je dis "vous écrivez juste une petite phrase pour raconter votre weekend" ou quoi, et s'ils ne savent pas écrire le mot, bin ils sont bloqués. Tu vois, alors qu'ils savent l'écrire phonétiquement. Alors j'ai beau dire l'orthographe, c'est pas grave, on fera ça après, ce qui m’importe, c'est que vous écriviez une phrase. [...] S'il y en a un qui écrit horloge justement, un autre élève va mettre que c'est faux et... Ils vont tous venir me trouver "quest-ce qu'il a écrit?" " ( $5^{\mathrm{e}}$ entretien). Ce concept de blocage amène l'enseignante à proposer peu d'activités de ce type et à demander aux enfants de changer de mot lorsqu'ils ne savent pas comment l'orthographier. Il la conduit également à proposer des tâches de production écrite très simples : «C'est parce que c'est quelque chose qu'ils savent bien écrire, tout simplement. Écrire son prénom [...] pour ne pas qu'ils soient freinés par l'orthographe [...] Parce que, eux, quand ils n'arrivent pas à écrire un mot, quand ils ne savent pas exactement comment ça s'écrit, parfois ils sont stressés » ( $5^{\mathrm{e}}$ entretien). 
2. Peut-on identifier, dans ce modèle, des leviers ou, au contraire, des freins conceptuels à un enseignement efficace de la lecture?

Les activités d'enseignement observées semblent régies à la fois par des concepts pragmatiques et pragmatisés qui, bien que décrits un à un dans cet article, fonctionnent en réseau. Alors que les premiers sont le fruit d'une élaboration personnelle dans l'action du sujet telle que définie par Pastré (2011); les seconds résultent d'une transformation de concepts théoriques qui, devenant des outils pour la conduite de l'activité, acquièrent un statut de connaissances pour l'action.

Les concepts de «blocage » et de «drill» ne semblent faire référence à aucun concept scientifique ou technique. Nous pourrions dès lors faire l'hypothèse de leur caractère purement pragmatique. Ils auraient donc été entièrement élaborés par le sujet. En revanche, les deux concepts organisateurs «maitrise du processus d'inférence » et «connaissance des sons» s'apparentent davantage à des concepts pragmatisés probablement issus de connaissances théoriques qui, en devenant opératives, acquièrent une nouvelle signification. Ainsi, le concept de connaissance des sons semble être issu du concept scientifique de correspondance grapho-phonémique. Ici, le concept diffère de la notion initiale et est simplifié au niveau de la terminologie (notion de «son » employée de manière récurrente en lieu et place du terme scientifique " phonème »).

Par ailleurs, le terme d'inférence, peu commun dans le vocabulaire des non-initiés, semble indiquer que le concept de « maitrise du processus d'inférence » qui organise l'action de l'enseignante est issu du concept scientifique d'inférence. Dans les prescrits (Socles de compétences) de Belgique francophone, ce concept est défini comme la mise « en rapport [de] deux ou plusieurs éléments d'un document entre eux $[. .$.$] pour construire une signification qui n'est pas explicitement donnée dans le texte » (Ministère$ de la Communauté française, 1999, p. 21). Mobilisé dans l'action, le concept d'inférence est donc redéfini par l'enseignante. Pour elle, comme énoncé lors de l'entretien qui suit la deuxième observation, l'inférence consiste à « remettre le mot dans son contexte et le comprendre, par rapport au contexte dans lequel il est. Et donner du sens quoi.» Ainsi, une fois opérationnalisé par l'enseignante, le concept est centré sur certains exemples-clés (les mots-indices, les pronoms personnels...). Par ailleurs, les observations et les entretiens ont montré que l'enseignante semblait privilégier des processus d'inférence textuelle. Julie ne semblait pas mettre l'accent sur les démarches d'inférence impliquant des informations présentes sur des images.

Ces concepts sont-ils en adéquation avec les recommandations issues de méta-analyses comme celle de Higgins et al. (2016)?

Le sujet semble axer son action vers un enseignement explicite de la compréhension. Cela s'observe dans ses pratiques et se retrouve notamment dans la notion de « mot-indice » qu'elle a défini avec ses élèves. Cela nous permet de faire l'hypothèse d'une volonté d'enseigner la compréhension en rendant visibles certains processus de compréhension selon une démarche qui s'approche d'un travail métacognitif.

Par ailleurs, le concept de « connaissance des sons » semble se concrétiser dans l'action par l'utilisation de la lecture oralisée à des fins diagnostiques, ce qui peut également constituer un levier efficace pour l'apprentissage de la lecture-écriture. 
En revanche, il semble que certains aspects du modèle opératif de l'enseignante freinent le recours aux activités de production écrite pour enseigner la lecture. Pour elle, l'un est simplement l'outil d'évaluation de l'autre. Par ailleurs, le concept de blocage l'amène à éviter ou à circonscrire les activités d'écriture à des tâches de réponse à des questions. Les indicateurs que l'enseignante prend dans l'action la conduisent à poser comme diagnostic que les élèves sont « stressés " à l'idée de mal orthographier un mot. Ce concept de blocage semble lié à la croyance qu'il faut préserver les élèves et ne pas les placer dans des tâches d'écriture qui les amènent à écrire des mots dont ils ne connaissent pas l'orthographe. Il pourrait être intéressant de déconstruire cette croyance et de lui donner les outils afin de pouvoir aider les enfants à surmonter cette difficulté d'encodage en expérimentant par exemple l'usage de référentiels et le tâtonnement.

\section{Discussion}

L'analyse du modèle opératif de cette enseignante donne à voir une certaine singularité dans les modes d'enseignement/apprentissage de la lecture. Que les savoirs d'action et les variables de l'environnement de classe utilisés pour guider son action diffèrent selon les professionnels parait logique dans un métier dont les caractéristiques des tâches prescrites sont aussi peu définies. En revanche, le rôle souvent inconscient joué par ces concepts pragmatiques ou savoirs d'action nous semble souvent ignoré en formation continue faute de temps accordé à l'analyse de l'activité en contexte. Des travaux tels que ceux de Huart (2010, p. 91) montrent qu'il est néanmoins possible « en partant de pratiques initiales et de représentations liées à ces pratiques " de susciter une modification des modèles opératifs qui intègre d'une part « des apports de connaissances » théoriques nouvelles et, d'autre part, « l'intégration de savoirs professionnels et d'expérience rendus possibles par les interventions des professionnels et par les interactions au sein du groupe ». Cadre d'analyse trop peu exploité dans le monde de la formation des enseignants, la didactique professionnelle vise à optimiser les apports de l'expérience, tout en respectant l'identité du professionnel et les spécificités des situations.

\section{Limite de la recherche}

Bien que les démarches d'analyse utilisées aient été choisies notamment pour objectiver l'analyse du chercheur, nous n'avons pas mis en place, dans le cadre de l'analyse de contenu, une technique visant à éprouver la pertinence des catégories formulées par le chercheur, de type codage inversé (Van der Maren, 2001, cité dans Richard, 2006), par exemple. Il est donc possible que ces catégories ne soient pas tout à fait exemptes de la sensibilité du chercheur.

\section{Perspectives de recherche}

Il nous semble intéressant de mettre en place des recherches du même type que la présente, concernant également l'enseignement initial de la lecture, mais en intégrant l'analyse de l'effet produit par la mise à jour des concepts organisateurs qui régissent l'activité des enseignants sur l'évolution de leur activité.

Parallèlement, à défaut de constituer une véritable structure conceptuelle liée à l'enseignement de la lecture, puisque l'élaboration d'une telle structure semble difficile dans le cadre du métier d'enseignant (Vinatier, 2013), il serait intéressant d'étudier la pragmatisation sur le long terme de certains concepts 
scientifiques par les professionnels pour nourrir la réflexion sur l'enseignement de ces concepts en formation initiale et continue.

\section{Notes}

1 Notre choix s'est porté sur celui pour lequel nous avons pu dégager le plus d'informations quant au modèle opératif. En d'autres termes, celui dont le modèle opératif est le plus étayé.

2 Nous avons en effet observé que les quatre sujets initiaux recouraient régulièrement à des moments d'écriture dans des leçons de lecture (exercices, questions avec réponses par écrit, etc.).

3 Cette notion de « mot-indice » qu'elle a développée avec ses élèves désigne les mots du texte qui permettent d'inférer une information.

4 Le terme « inférence » renvoie à un concept scientifique qui diffère du sens que lui attribue l'enseignante. Dans la suite des résultats, nous reviendrons sur ce point.

\section{Références}

Clot, Y. et Leplat, J. (2005). La méthode clinique en ergonomie et en psychologie du travail. Le travail humain, 68(4), 289316. http://dx.doi.org/10.3917/th.684.0289

Goigoux, R. (2007). Un modèle d'analyse de l'activité des enseignants. Éducation et didactique, 1(3), 47-69. http://dx.doi.org/10.4000/educationdidactique.232

Goigoux, R. et Cèbe, S. (2009). Un autre rapport entre recherche, pratique et formation. Les instruments didactiques comme vecteur de transformation des pratiques des enseignants confrontés aux difficultés d'apprentissage des élèves. Dans Colloque du Réseau international de recherche en éducation et formation (REF) (p. 1-11). Repéré à http://halshs.archives-ouvertes.fr/halshs-00936348

Goigoux, R., Jarlégan, A. et Piquée, C. (2015). Évaluer l'influence des pratiques d'enseignement du lire-écrire sur les apprentissages des élèves : enjeux et choix méthodologiques. Recherches en didactiques, 1(19), 9-37.

Higgins, S., Henderson, P., Martell, T., Sharples, J. et Waugh, D. (2016). Improving literacy in key stage one: Guidance report. Repéré à https://educationendowmentfoundation.org.uk/public/files/Publications/Campaigns/Literacy/KS1 Literacy Guidance.pdf

Huart, V. (2010). L'intérêt de la didactique professionnelle pour la mise en œuvre d'une pragmatique de formation. Savoirs, 23, 73-94. http://dx.doi.org/10.3917/savo.023.0073

L'Écuyer, R. (1987). L'analyse de contenu : notions et étapes. Dans J.-P. Deslauriers (dir.), Les méthodes de la recherche qualitative (p. 49-65). Sillery, QC : Presses de l'Université du Québec.

Lefeuvre, G. et Murillo, A. (2017). Évolution de l'activité d'enseignement au cours de l'année : analyse à partir de la théorie de la conceptualisation dans l'action. Éducation et didactique, 11(3), 73-100. http://dx.doi.org/10.4000/educationdidactique.2845

Leplat, J. (2011). Mélanges ergonomiques : activité, compétence, erreur. Toulouse : Octarès.

Ministère de la Communauté française. (1999). Socles de compétences. Bruxelles : Administration générale de l'enseignement et de la recherche scientifique.

Mullis, I. V. S., Martin, M. O., Foy, P. et Hopper, M. (2017). PIRLS 2016: International results in reading. Repéré à https://eric.ed.gov/?id=ED580353

Pastré, P. (2011). La didactique professionnelle : un point de vue sur la formation et la professionnalisation. Education Sciences É Society, 2(1), 83-95. Repéré à https://riviste.unimc.it/index.php/es s/article/view/136

Pastré, P. (2013). La didactique professionnelle : approche anthropologique du développement chez les adultes. Paris : Presses universitaires de France. 
Pastré, P., Mayen, P. et Vergnaud, G. (2006). La didactique professionnelle. Revue française de pédagogie, (154), 145-198. http://dx.doi.org/10.4000/rfp.157

Piaget, J. (1974). Réussir et comprendre. Paris : Presses universitaires de France.

Prost, A. (2001). Pour un programme stratégique de recherche en éducation. Repéré à http://media.education.gouv.fr/file/93/4/5934.pdf

Rappe, J. (2015). Quelle diversité conceptuelle peut-on observer dans les modèles opératifs de quatre enseignants de deuxième année primaire dans le cadre de l'enseignement de la lecture? (Mémoire de master inédit). Université de Liège.

Richard, S. (2006). L'analyse de contenu pour la recherche en didactique de la littérature. Le traitement de données quantitatives pour une analyse qualitative : parcours d'une approche mixte. Recherches qualitatives, 26(1), 181-207. Repéré à http://www.recherche-qualitative.qc.ca/documents/files/revue/edition reguliere/numero26(1)/srichard ch.pdf

Schillings, P., Dupont, V., Géron, S. et Matoul, A. (2017). PIRLS 2016 : Note de synthèse. Repéré à http://hdl.handle.net/2268/216693

Schillings, P., Géron, S. et Dupont, V. (2017). Étude comparative des pratiques d'enseignement de la lecture en $4^{\mathrm{e}}$ primaire : des questions de didactique pointées par l'étude internationale PIRLS 2011. Forumlecture suisse, 2017(3). Repéré à http://hdl.handle.net/2268/215725

Schillings, P., Hindryckx, G., Dupont, V., Matoul, A. et Lafontaine, D. (2012). PIRLS 2011. Enquête internationale sur le développement des compétences en lecture des élèves de $4^{e}$ année primaire. Note de synthèse. Repéré à http://www.enseignement.be/download.php?do id=9687\&do check=

Schillings, P. et Lafontaine, D. (2013). Les résultats de l'enquête PIRLS sur la lecture en quatrième année primaire : des compétences à mettre à l'étude. Caractères, 44, 5-15. Repéré à https://orbi.uliege.be/handle/2268/145460

Vergnaud, G. (1996). Au fond de l'action, la conceptualisation. Dans J.-M. Barbier (dir.), Savoirs théoriques et savoirs d'action (p. 275-292). Paris : Presses universitaires de France.

Vermersch, P. (2004). Aide à l'explicitation et retour réflexif. Éducation permanente, 160(numéro spécial : Analyse des pratiques), 71-80.

Vinatier, I. (2013). Le travail de l'enseignant. Une approche par la didactique professionnelle. Bruxelles : De Boeck.

\section{Pour citer cet article}

Rappe, J., Schillings, P., Depluvrez, Y. et Dejaegher, C. (2018). Analyse du modèle opératif d'un enseignant en deuxième année primaire dans le cadre de l'enseignement de la lecture. Formation et profession, 26(3) 81-93. http://dx.doi.org/10.18162/fp.2018.475 